\title{
Maternal excess gestational weight gain and infant waist circumference: a 2-y observational study
}

\author{
Sara F. Michaliszyn', Lindsey A. Sjaarda², Christina Scifres ${ }^{3}$, Hyagriv Simhan ${ }^{4}$ and Silva A. Arslanian ${ }^{5}$
}

BACKGROUND: The purpose of this study was to examine the effects of gestational weight gain (GWG) and infant feeding practices on infant growth parameters in infants from 6 to 24 mo of age.

METHODS: Forty mother-infant pairs were recruited after delivery and followed up to 24 mo postpartum. GWG was calculated as prepregnancy weight subtracted from weight at delivery. Infant weight velocity was calculated as the change in weight between consecutive visits divided by the intervening time. Infant feeding practices were measured by interview and infant growth and waist circumference by standard anthropometry.

RESULTS: Infants born to mothers with excess GWG were heavier at birth $(3,521 \pm 91$ vs. 3,196 $\pm 97 \mathrm{~g}, P=0.02)$ and had an average $2.16 \pm 1.1 \mathrm{~cm}(P=0.03)$ larger waist circumference throughout the 24 mo compared with infants born to mothers with appropriate GWG. Waist circumference increased by 0.12 and $2.0 \mathrm{~cm}$ for every 1 unit increase in GWG and infant birth weight.

CONCLUSION: Infants born to women who exceeded the Institute of Medicine (IOM)-recommended guidelines for GWG were heavier at birth and had a significantly higher waist circumference up to 2 y of age. Strategies to control maternal excess GWG and thus the outcome on infant birth weight and waist circumference should be pursued.

0 verweight and obesity begin as early as infancy with $8.1 \%$ of infants and toddlers (birth - age $24 \mathrm{mo}$ ) exceeding the CDC sex-specific weight for recumbent length growth charts (1). Parallel to the increasing prevalence of childhood obesity, obesity-related comorbidities such as insulin resistance and systemic inflammation are observed in children as young as birth (2). Factors underlying this phenomenon are complex although suboptimal prenatal and/or early postnatal growth may play a role in the development of obesity and obesityassociated comorbidities early in life $(3,4)$. Indeed, maternal factors, such as a high prepregnancy BMI and excessive gestational weight gain (GWG) make critical contributions to increases in adiposity in infancy $(4-6)$, childhood $(2,7)$, adolescence (8), and early adulthood $(9,10)$.
The period of infancy (birth - age $24 \mathrm{mo}$ ) may be critical to long-term health, but few studies have examined both the role of in utero and early feeding practices on infant growth and development. The preponderance of the evidence suggests that formula-feeding increases the rate of infants' weight gain up to the first year of life $(4,11)$ but may not persist into the second year of life (12). However, data describing the association between maternal obesity and GWG in combination with infant feeding practices (breast vs. formula) on infant growth and adiposity up to $24 \mathrm{mo}$ of age are lacking. Therefore, as part of a pilot study of infant growth and adiposity, we measured infant weight, length, waist circumference, and skinfold thickness between 6 and 24 mo of age in infants born to mothers with appropriate (AGWG) vs. excess GWG (EGWG). Using mixed-effects modeling, we hypothesized that infants born to mothers with EGWG would weigh more and have a larger waist circumference throughout the observational period compared with infants born to mothers with AGWG. In addition, we assessed postnatal infant feeding methods in combination with GWG and their relationship with infant growth rate and adiposity between 6 and 24 mo of age.

\section{RESULTS}

\section{Maternal and Infant Characteristics}

Maternal characteristics are presented in Table 1. Of the 40 total participating mothers, 20 women were normal weight $\left(\mathrm{BMI}<25 \mathrm{~kg} / \mathrm{m}^{2}\right)$ and 20 were overweight to obese (BMI $\geq$ $\left.25 \mathrm{~kg} / \mathrm{m}^{2}\right)$ at preconception. Mothers were of comparable age (overall mean \pm SEM, 29.1 \pm 0.7 ), primarily of Caucasian race and nonsmoking. There were no differences in infant race or gender distribution between groups. Most infants were formula fed up to 12 mo of age although the proportion of formula fed vs. breast fed infants were statistically similar between AGWG and EGWG (Chi-square, $P>0.05$ ) (Table 1).

\section{Longitudinal Changes in Maternal and Infant Characteristics}

At $6,12,18$, and 24 mo postpartum, women with EGWG had significantly higher BMI and waist circumference compared with women with AGWG (Table 2). Moreover, infants born to mothers with EGWG vs. AGWG had significantly higher birth weights $(3,521 \pm 91$ vs. $3,196 \pm 97 \mathrm{~g}, P=0.02)$, with larger

'Human Performance and Exercise Science, Youngstown State University, Youngstown, Ohio; ${ }^{2}$ Eunice Kennedy Shriver National Institute of Child Health and Human

Development, Rockville, Maryland; ${ }^{3}$ College of Medicine, The University of Oklahoma, Norman, Oklahoma; ${ }^{4}$ Division of Maternal-Fetal Medicine, University of Pittsburgh School of Medicine, Pittsburgh, Pennsylvania; ${ }^{5}$ Division of Weight Management and Wellness and Division of Pediatric Endocrinology, Metabolism \& Diabetes Mellitus, Children's

Hospital of Pittsburgh, Pittsburgh, Pennsylvania. Correspondence: Sara F. Michaliszyn (sbmichaliszyn@ysu.edu)

Received 28 March 2016; accepted 7 July 2016; advance online publication 5 October 2016. doi:10.1038/pr.2016.174 
average waist circumference $(2.16 \pm 1.1 \mathrm{~cm}$ higher, $P=0.03)$ from 6 mo to 2 y (Figure 1). This difference remained even when accounting for differences in prepregnancy BMI but lost significance after accounting for the differences in infant birth weight. There were no differences detected in weight, length, weight-for-length, ponderal index, weight velocity, or sum of skinfolds in infants born to mothers exceeding or within GWG guidelines (Table 2). Likewise, there were no differences detected in weight velocity, ponderal index, waist circumference, sum of skinfolds, or weight-for-length between formula fed and breast fed infants (data not shown).

Table 1. Maternal and infant characteristics

\begin{tabular}{lccc}
\hline Maternal characteristics & & & T-test, \\
Variable & AGWG & EGWG & $P$ \\
\hline$N$ & 21 & 19 & \\
Age (years) & $30.2 \pm 0.9$ & $27.8 \pm 1.1$ & NS \\
Race (\% white) & $76 \%$ & $79 \%$ & NS \\
Smoking at 6 mo (\% yes) & $31 \%$ & $24 \%$ & NS \\
SES (\% > \$50,000) & $44 \%$ & $40 \%$ & NS \\
GWG (kg) & $10.1 \pm 1.2$ & $17.6 \pm 1.0$ & $<0.001$ \\
NW/OB (N) & $15 / 6$ & $5 / 14$ & 0.004 \\
Prepregnancy BMI (kg/m²) & $24.3 \pm 1.4$ & $29.9 \pm 1.5$ & 0.01 \\
Infant characteristics & & & \\
Race (\% white) & $71 \%$ & $68 \%$ & NS \\
Gender (\%Male) & $61 \%$ & $42 \%$ & NS \\
Gestational age (weeks) & $39.8 \pm 0.3$ & $39.6 \pm 0.3$ & NS \\
Birth weight (g) & $3,176.1 \pm 77.1$ & $3,485.7 \pm 118.5$ & 0.03 \\
Formula/breast fed/none 6 mo (\%) & $67 / 33 / 0 \%$ & $88 / 12 / 0 \%$ & NS \\
Formula/breastfed/none 12 mo (\%) & $58 / 31 / 11 \%$ & $56 / 31 / 13 \%$ & NS \\
Formula/breastfed/none 18 mo (\%) & $0 / 13 / 87 \%$ & $5 / 18 / 77 \%$ & NS \\
Formula/breast fed/none 24 mo (\%) & $0 / 0 / 100 \%$ & $0 / 15 / 85 \%$ & NS \\
\hline
\end{tabular}

Mean \pm SEM, SES, socioeconomic status; GWG, gestational weight gain; NW, normal weight $\left(\mathrm{BMl}<25 \mathrm{~kg} / \mathrm{m}^{2}\right)$; OB, obese $\left(\mathrm{BMl}>25 \mathrm{~kg} / \mathrm{m}^{2}\right)$.

\section{Associations With Infant Anthropometrics}

In an effort to examine the determinants of the larger average waist circumference observed from 6 to 24 mo in infants born to EGWG mothers, multiple linear regression models were used (Table 3). Infant waist circumference increased $0.18 \mathrm{~cm}$ for every 1 unit increase in maternal GWG. When infant birth weight was included in the model, the parameter estimate for maternal GWG decreased by $\sim 31 \%$. Accordingly, waist circumference increased $2.0 \mathrm{~cm}$ for every 1 unit increase in infant birth weight and by $0.12 \mathrm{~cm}$ for every 1 unit increase in GWG (Table 3). When the categorical variable of infant feeding practice was entered into the model (model 3, Table 3), the parameter estimate for maternal GWG remained unchanged and no independent contribution in feeding practices was observed. Maternal prepregnancy BMI did not significantly contribute to the variability in infant waist circumference.

\section{DISCUSSION}

The results of this study suggest that, independent of pregravid weight, women who gained in excess of the 2009 IOM recommended GWG gave birth to infants who were $9 \%$ heavier at birth and had an average 5\% larger waist circumference through infancy (6 mo to $2 \mathrm{y}$ of age), compared with infants born to mothers who gained within the 2009 IOM guidelines. The present investigation further demonstrates that maternal GWG, and consequently infant birth weight, and to a lesser extent infant feeding practices, appear to play a larger role in an infant precursor of central adiposity (i.e., waist circumference) up to $2 \mathrm{y}$ of age.

Childhood obesity is suggested to be set by both early prenatal and postnatal influences. High prepregnancy BMI and excess GWG increase the risk for large-for-gestational-age offspring and obesity across the lifespan. Starling et al. estimated associations between maternal BMI and GWG throughout pregnancy and demonstrated that a $0.1 \mathrm{~kg} /$ week increase in predicted GWG associated with increased fat mass, fat free mass, and percent body fat at birth (6). Increased adiposity at birth may predispose these children to obesity later in life. Indeed,

Table 2. Maternal and infant anthropometric measures over time, according to GWG status

\begin{tabular}{|c|c|c|c|c|c|c|c|c|c|}
\hline & \multicolumn{2}{|c|}{$6 \mathrm{mo}$} & \multicolumn{2}{|c|}{$12 \mathrm{mo}$} & \multicolumn{2}{|c|}{$18 \mathrm{mo}$} & \multicolumn{2}{|c|}{$24 \mathrm{mo}$} & \multirow{2}{*}{$\frac{\text { Repeated measures }}{P}$} \\
\hline & AGWG & EGWG & AGWG & EGWG & AGWG & EGWG & AGWG & EGWG & \\
\hline \multicolumn{10}{|l|}{ Maternal } \\
\hline Waist (cm) & $89.4 \pm 3.9$ & $96.1 \pm 4.5$ & $85.5 \pm 4.3$ & $96.3 \pm 3.3$ & $83.2 \pm 3.7$ & $98.6 \pm 3.9$ & $94.0 \pm 21.5$ & $103.2 \pm 4.7$ & 0.005 \\
\hline \multicolumn{10}{|l|}{ Infant } \\
\hline Length $(\mathrm{cm})$ & $67.7 \pm 0.6$ & $67.7 \pm 0.7$ & $74.8 \pm 0.7$ & $75.0 \pm 0.5$ & $81.6 \pm 1.1$ & $82.4 \pm 0.6$ & $85.0 \pm 2.3$ & $88.2 \pm 1.1$ & NS \\
\hline Weight-for-length & $49.7 \pm 8.4$ & $65.6 \pm 10.8$ & $69.6 \pm 6.3$ & $76.6 \pm 5.2$ & $69.1 \pm 7.4$ & $70.9 \pm 6.6$ & $44.9 \pm 15.1$ & $70.3 \pm 8.0$ & NS \\
\hline $\mathrm{PI}\left(\mathrm{kg} / \mathrm{m}^{3}\right)$ & $25.3 \pm 0.6$ & $25.7 \pm 0.8$ & $23.8 \pm 0.4$ & $24.0 \pm 0.5$ & $21.0 \pm 0.6$ & $20.2 \pm 0.8$ & $18.4 \pm 0.6$ & $17.9 \pm 0.8$ & NS \\
\hline Weight velocity (g/mo) & $780.6 \pm 47.9$ & $760.7 \pm 45.8$ & $345.7 \pm 36.7$ & $342.8 \pm 38.0$ & $274.5 \pm 29.5$ & $230.9 \pm 24.7$ & $228.0 \pm 36.2$ & $138.1 \pm 53.1$ & NS \\
\hline
\end{tabular}

Mean \pm SEM. 
retrospective, epidemiological investigations have demonstrated an association between excess GWG and offspring obesity in adolescents $(8)$ and adulthood $(9,10)$; however, little exploration has been dedicated to examining the interrelated effects of maternal GWG and infant feeding practices on infant anthropometrics during the first 2 y of life. Our findings support previous findings, and demonstrate infants born to mothers exceeding the IOM guidelines were significantly heavier at birth and this remained upon adjustment for prepregnancy BMI. Furthermore, we observed a marked difference in waist circumference which may have been present starting at birth. This difference may be likely to persist, as prior data from a prospective birth cohort study, conducted from 1998 to 2013 in African American and Dominican dyads, demonstrated a

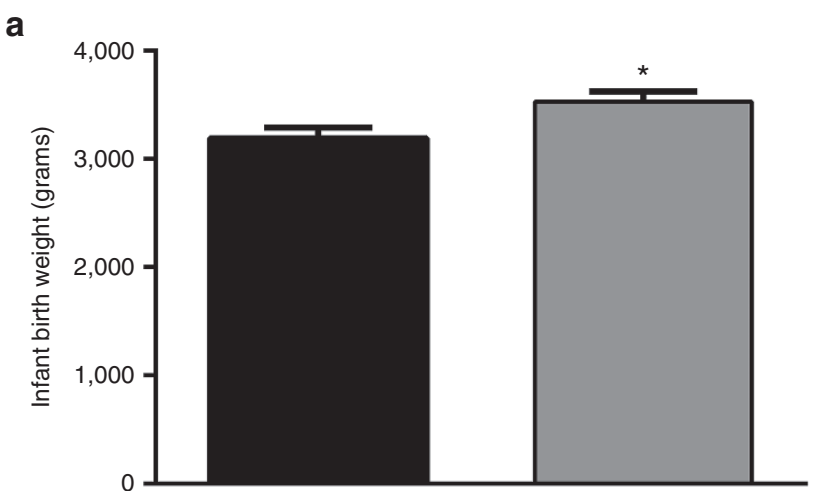

b

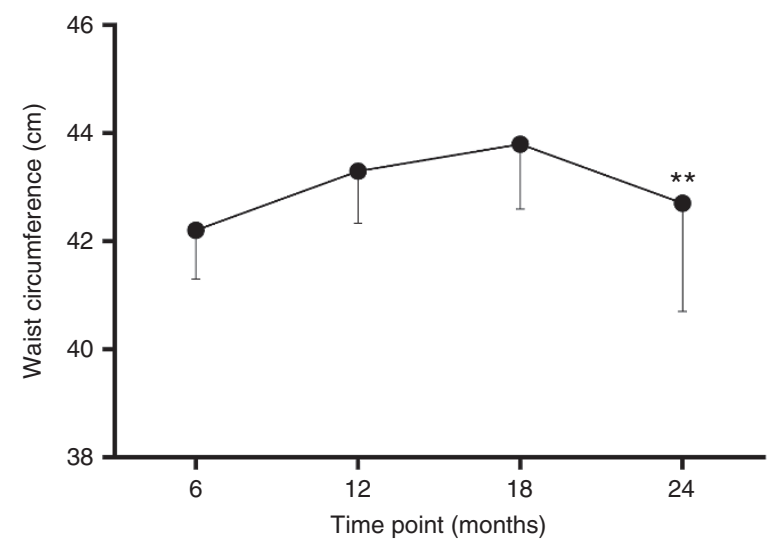

Figure 1. Infant birth weight (a), and infant waist circumference (b) in infants born to mothers with AGWG (black fill; $N=21$ ) and EGWG (gray fill; $N=19$ ). Plots are mean \pm SEM. ${ }^{*} P<0.02 ;{ }^{* *} P<0.03$ using mixed-effects modeling of repeated-measures data. positive association between excessive GWG and offspring waist circumference at $7 \mathrm{y}$ of age (13). In a separate retrospective analysis examining 6,837 mother-child dyads, $15.1 \%$ of children (mean age 5.8 y) presented with increased abdominal adiposity in mothers that gained weight excessively (14). Our findings extend these observations and demonstrate that infants born to mothers exceeding the IOM guidelines weigh more at birth and exhibit an increased waist circumference as early as 6 mo of age. In youth, waist circumference can be used as an indicator of disease risk, as abdominal obesity is associated with cardiovascular risk factors such as hyperinsulinemia, lipid and lipoprotein concentrations, and blood pressure (15-17). The difference in waist circumference in infants born to EGWG mothers raises the possibility of an increase in central adiposity which may reflect an increased metabolic risk in these infants. The determinants of waist circumference and cardiometabolic risk at this early stage of life, as well as the physiological causes, remain to be investigated. It is well established, however, that infancy is a critical period of development, and further studies are needed to establish whether interventions may help change the trajectory of relevant risk factors for obesity in the early period after birth.

There is a particular interest in understanding the relationship between infant feeding practices and infant growth due to the observed differences in infant weight gain between breastand formula-fed infants. Compared with breast-fed infants, formula-fed infants are shown to gain weight faster toward the end of the first year of life $(4,18)$. On the other hand, Butte et al. found that though infants exclusively formula-fed for the first 4 mo of life gained weight faster at 3-6 mo of age, these differences did not persist into the second year of life (12). Kramer et al. extended these observations to $6.5 \mathrm{y}$ in 13,889 infants participating in the Promotion of Breastfeeding Intervention Trial (PROBIT) and did not find an intervention effect on measures of adiposity, increased stature, or reduced blood pressure (19). Similar to these studies, our data show that infant feeding practices did not significantly contribute to the variability in infant anthropometrics or waist circumference up to $2 \mathrm{y}$ of age. Although we did not find any significant differences in infant weight gain, further investigations need to clarify the relationship between maternal GWG and timing of infant feeding practices on infant growth.

A limitation of this study is that while GWG contributed to infant birth weight and waist circumference up until $2 \mathrm{y}$ of age, we do not know the timing of GWG which could contribute

Table 3. Independent predictors of infant waist circumference

\begin{tabular}{|c|c|c|c|c|c|c|c|c|c|}
\hline \multirow[b]{2}{*}{ Variable } & \multicolumn{3}{|c|}{ Model 1} & \multicolumn{3}{|c|}{ Model 2} & \multicolumn{3}{|c|}{ Model 3} \\
\hline & B & SE B & $\beta$ & B & SE B & $\beta$ & B & SE B & $\beta$ \\
\hline GWG & 0.18 & 0.06 & $0.313^{*}$ & 0.12 & 0.06 & $0.215^{* *}$ & 0.18 & 0.06 & $0.296^{* *}$ \\
\hline Infant Feeding practices & & & & & & & -0.565 & 0.50 & -0.110 \\
\hline$R^{2}$ & & 0.10 & & & 0.14 & & & 0.10 & \\
\hline
\end{tabular}

${ }^{*} P=0.001$. ${ }^{*} P<0.05$. 
further to a more nuanced understanding of the effects of GWG on infant growth. Women who gain excess weight during early pregnancy are shown to have larger infants at birth compared with women who gain during late gestation $(6,20)$. Future studies should be directed toward understanding the underlying effects and processes of timing of GWG on infant adiposity development. Due to the nature of the pilot study, not every mother-infant pair was enrolled in the study from birth on. This allowed for nonequivalent mother-infant pairs during each subsequent time point possibly reducing power to definitively determine any modulating effect of feeding practices on infant weight gain.

In conclusion, infants born to mothers who exceeded the IOM recommended guidelines for GWG were heavier at birth and had a significantly higher waist circumference up to $2 \mathrm{y}$ of age. As infant birth weight explained a significant proportion of the variability in infant waist circumference, strategies to control maternal excess GWG and consequently the outcome on infant birth weight and waist circumference should be more closely studied with adequate measurement of central adiposity measures along with conventional growth measurements.

\section{METHODS}

The Healthy Infants Postpartum (HIP) study was a pilot observational study of infant growth and adiposity in infants 6-24 mo of age, born to mothers spanning the range of BMI and GWG (2011 to 2013). Research was conducted in accord with prevailing ethical principles and the University of Pittsburgh Institutional Review Board approval was obtained, and parental consent was obtained from each mother for herself and her child prior to study participation.

\section{Subjects}

Participants for the HIP study $(n=40)$ were recruited through direct mailings to women who were prior nulliparous participants with singleton pregnancies in the Study of Nutrition and Pregnancy (SNAP) and the Nulliparous Pregnancy Outcomes Study: Monitoring Mothers-to-be (nuMoM2b) (Clinical Trial Number: NCT01322529) at University of Pittsburgh, Pittsburgh PA, USA. Women were eligible for inclusion if they had delivered at $\geq 370 / 7 \mathrm{wk}$ gestation and if they did not have pregestational diabetes, gestational diabetes, pre-eclampsia or gestational hypertension, chronic hypertension, or rheumatologic disorders during their pregnancy. Women were also excluded if they were taking chronic steroid therapy, as this could have had effects on neonatal growth and development. Mother-infant pairs with known fetal anomalies (e.g., congenital heart defects) were also excluded from the study.

Study visits were conducted after birth every 6 mo (i.e., 6 mo, 12 mo, $18 \mathrm{mo}$, and $24 \mathrm{mo}$ ) beginning as early as 6 mo of age. Mother-infant pairs were invited to the first visit where they could be contacted, and this resulted in study visits beginning at 6 mo of age in 22 infants $(55 \%)$, at $12 \mathrm{mo}$ of age in 13 infants (33\%), and $18 \mathrm{mo}$ of age in 5 infants (12\%). Follow-up visits were scheduled every 6 mo to a maximum age of 24 mo through the 2 -y study period which resulted in a total of 22 visits at $6 \mathrm{mo}, 32$ at $12 \mathrm{mo}, 31$ at $18 \mathrm{mo}$, and 15 at $24 \mathrm{mo}$.

\section{Maternal and Infant Anthropometry}

Maternal standing height and weight were measured to the nearest $\mathrm{cm}$ and $\mathrm{kg}$, respectively, at each study visit. Waist circumference was assessed using duplicate measurements obtained with a spring-loaded Gulick tape. Triplicate measures were obtained if two measures were $>5 \mathrm{~mm}$ apart. Participants stood with arms at sides, feet together and abdomen relaxed; waist circumference was measured at the level of the iliac crest, usually at the level of the umbilicus. Prepregnancy BMI was calculated from weight and height data measured between
$8-13$ wk of gestation from the prior study record (SNAP, nuMoM2b), and information about general maternal health during gestation was obtained from the parent studies. At each study visit, infant length was measured using an infantometer (Seca 416 Infantometer), weight using an electronic infant scale, and waist circumference just above the plane connecting the superior iliac crests, with the infant lying down. Skinfold thickness was measured in triplicates using Lange skinfold calipers in four locations (bicep, triceps, subscapular, suprailiac) (21) and the mean skinfold measurement was calculated for each location.

\section{Infant Feeding Practices}

Due to the nature of the pilot study, infant feeding questions were adapted from the Infant Feeding Practices Study II (IFPS II) questionnaires, at each study visit (http://www.cdc.gov/breastfeeding/data/ ifps/questionnaires.htm). The mothers were asked about their current infant feeding practices including infants' current milk consumption and the proportion of milk from breast or formula, and whether infants consumed breast milk from the breast (vs. from a bottle). Mothers who reported any proportion of milk derived from breast milk were classified as breastfeeding for purposes of this analysis.

\section{Calculations}

GWG was calculated as weight at delivery minus weight measured between $8-13$ wk of gestation. The 2009 IOM guidelines were used to classify GWG as excessive vs. not excessive (22). Gestational age at delivery was obtained from the parent studies, for which the gestational age was determined by the best clinical date available during pregnancy as assigned by the treating obstetricians. Infant weight velocity was calculated as the difference in weight measured between study visits divided by the number of intervening months. Ponderal index (PI) was calculated as weight/length $\left(\mathrm{cm}^{3}\right)$ multiplied by 100 .

\section{Statistical Approach}

Baseline subject characteristics were compared using a Student $T$-test for quantitative variables and $\chi^{2}$ test for categorical variables between groups. ANCOVA models were used to assess between group differences adjusting for prepregnancy BMI and infant birth weight where necessary. Mixed effects modeling were used to examine longitudinal changes in infant growth parameters (weight-forlength, ponderal index, weight velocity, sum of skinfolds, and waist circumference) from 6 to 24 mo of age with adjustments for infant birth weight where necessary. Linear regression analyses were used to evaluate the association of GWG, infant birth weight, and infant feeding practices with infant growth parameters. Data are presented as mean \pm SEM. Statistical significance was set at $P \leq 0.05$ and the statistical analyses were performed using PASW Statistics (version 20, SPSS, Chicago, IL).

\section{ACKNOWLEDGMENTS}

We would like to thank all the mothers and their children who participated in this study, without whom science would not advance. We are grateful to the nursing and research staff of the parent studies, Study of Nutrition and Pregnancy (SNAP) and the Nulliparous Pregnancy Outcomes Study: Monitoring Mothers-to-be (nuMoM2b) (Clinical Trial Number: NCT01322529) for their attention towards subject recruitment.

\section{AUTHOR CONTRIBUTION}

S.F.M. first authored the manuscript, facilitated data collection, and analyzed and interpreted the data; L.A.S. and C.S. facilitated data collection and reviewed and edited the manuscript; H.S. supervised the study and critically reviewed/edited the manuscript; S.A. provided administrative, technical and material support, supervised the study, and critically reviewed/edited the manuscript.

\section{STATEMENT OF FINANCIAL SUPPORT}

This project was supported by Thrasher Research Fund.

Disclosure: None of the authors report any conflict of interest with respect to this work. 


\section{REFERENCES}

1. Ogden CL, Carroll MD, Kit BK, Flegal KM. Prevalence of childhood and adult obesity in the United States, 2011-2012. JAMA 2014;311:806-14.

2. Catalano PM, Presley L, Minium J, Hauguel-de Mouzon S. Fetuses of obese mothers develop insulin resistance in utero. Diabetes Care 2009;32:1076-80.

3. Brawarsky P, Stotland NE, Jackson RA, et al. Pre-pregnancy and pregnancy-related factors and the risk of excessive or inadequate gestational weight gain. Int J Gynaecol Obstet 2005;91:125-31.

4. Baker JL, Michaelsen KF, Rasmussen KM, Sørensen TI. Maternal prepregnant body mass index, duration of breastfeeding, and timing of complementary food introduction are associated with infant weight gain. Am J Clin Nutr 2004;80:1579-88.

5. Hull H, Baptiste-Roberts K, Salafia C, Brancati F, Gallagher D. Influence of gestational diabetes on the relationship between placental measures and BMI in early childhood. Obesity. 2008;16:S280-S280.

6. Starling AP, Brinton JT, Glueck DH, et al. Associations of maternal BMI and gestational weight gain with neonatal adiposity in the Healthy Start study. Am J Clin Nutr 2015;101:302-9.

7. Zilko CE. Economic contraction and birth outcomes: an integrative review. Hum Reprod Update 2010;16:445-58.

8. Oken E, Rifas-Shiman SL, Field AE, Frazier AL, Gillman MW. Maternal gestational weight gain and offspring weight in adolescence. Obstet Gynecol 2008;112:999-1006.

9. Reynolds RM, Osmond C, Phillips DI, Godfrey KM. Maternal BMI, parity, and pregnancy weight gain: influences on offspring adiposity in young adulthood. J Clin Endocrinol Metab 2010;95:5365-9.

10. Schack-Nielsen L, Michaelsen KF, Gamborg M, Mortensen EL, Sørensen TI. Gestational weight gain in relation to offspring body mass index and obesity from infancy through adulthood. Int J Obes (Lond) 2010;34:67-74.

11. Ziegler EE. Growth of breast-fed and formula-fed infants. Nestle Nutr Workshop Ser Pediatr Program 2006;58:51-9; discussion 59-63.

12. Butte NF, Wong WW, Hopkinson JM, Smith EO, Ellis KJ. Infant feeding mode affects early growth and body composition. Pediatrics 2000;106: 1355-66.
13. Widen EM, Whyatt RM, Hoepner LA, et al. Gestational weight gain and obesity, adiposity and body size in African-American and Dominican children in the Bronx and Northern Manhattan. Matern Child Nutr 2016; 12:918-28.

14. Ensenauer R, Chmitorz A, Riedel C, et al. Effects of suboptimal or excessive gestational weight gain on childhood overweight and abdominal adiposity: results from a retrospective cohort study. Int J Obes (Lond) 2013;37: 505-12.

15. Jago R, Mendoza JA, Chen T, Baranowski T. Longitudinal associations between BMI, waist circumference, and cardiometabolic risk in US youth: monitoring implications. Obesity (Silver Spring) 2013;21:E271-9.

16. Bassali R, Waller JL, Gower B, Allison J, Davis CL. Utility of waist circumference percentile for risk evaluation in obese children. Int J Pediatr Obes 2010;5:97-101.

17. Savva SC, Tornaritis M, Savva ME, et al. Waist circumference and waistto-height ratio are better predictors of cardiovascular disease risk factors in children than body mass index. Int J Obes Relat Metab Disord 2000;24:1453-8.

18. Dewey KG, Heinig MJ, Nommsen LA, Peerson JM, Lönnerdal B. Growth of breast-fed and formula-fed infants from 0 to 18 months: the DARLING Study. Pediatrics 1992;89(6 Pt 1):1035-41.

19. Kramer MS, Matush L, Vanilovich I, et al.; PROBIT Study Group. Effects of prolonged and exclusive breastfeeding on child height, weight, adiposity, and blood pressure at age $6.5 \mathrm{y}$ : evidence from a large randomized trial. Am J Clin Nutr 2007;86:1717-21.

20. Catov JM, Abatemarco D, Althouse A, Davis EM, Hubel C. Patterns of gestational weight gain related to fetal growth among women with overweight and obesity. Obesity (Silver Spring) 2015;23:1071-8.

21. Zemel BS, Riley EM, Stallings VA. Evaluation of methodology for nutritional assessment in children: anthropometry, body composition, and energy expenditure. Annu Rev Nutr 1997;17:211-35.

22. Institute of Medicine (US) and National Research Council (US) Committee to Reexamine IOM Pregnancy Weight Guidelines. Weight Gain During Pregnancy: Reexamining the Guidelines. In: Rasmussen KM, Yaktine AL, eds. Washington (DC): National Academies Press (US); 2009:1-3. 